\title{
A complication of a long-standing solitary lung cyst
}

\author{
JGH Hubbard, UU Nkere, R Azhar
}

A 56-year-old man, an ex-smoker, underwent a right middle lobectomy for an infected lung cyst. The cyst had been noted on a chest X-ray 34 years earlier (figure 1) although the patient had never previously had symptoms referable to it.

Six months prior to presentation the patient had developed an acute febrile illness with a cough productive of purulent sputum, intermittent haemoptysis, shortness of breath and weight loss. He subsequently improved on antibiotics. His chest X-ray showed that the cyst now had a fluid level (figure 1) and although the appearance improved on antibiotics, it failed to resolve completely. Investigations including bronchoscopy, sputum cytology and abdominal ultrasound examination were unremarkable. Computed tomography confirmed the chest X-ray features.

At operation, a consolidated middle lobe was found to be adherent to the upper and lower lobes and the chest wall. There was a large cyst measuring $7 \times 5 \mathrm{~cm}$ in the lateral portion of the lobe communicating with multiple loculi in the rest of the substance of the middle lobe. Macroscopically the lung was firm and there were several deposits of an irregular white tumour in the lung surrounding the cyst with slimy mucoid material present at the surface. The histology is shown in figure 2.

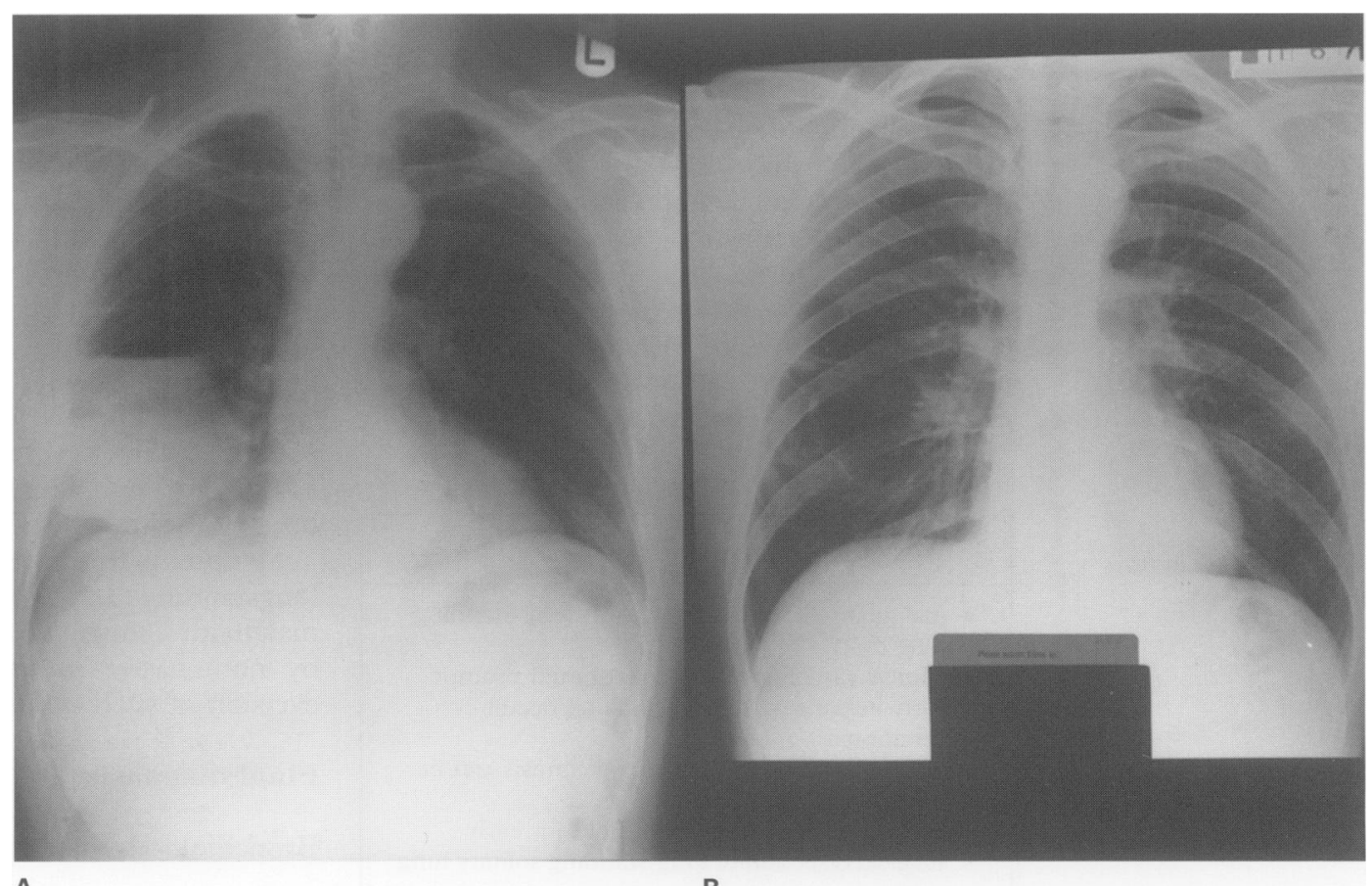

Freeman Hospital, Freeman Road, Newcastle upon Tyne, UK Department of Cardiothoracic Surgery JG Hubbard

UU Nkere

Department of Pathology

R Azhar

Correspondence to: Mr JGH Hubbard, Department of Surgery, The Medical School, University of Newcastle upon Tyne, Framlington Place, Newcastle upon Tyne NE2 4HH, UK

Accepted 9 December 1996
A

B

Figure 1 Chest $\mathrm{X}$-rays showing the solitary lung cyst in the right middle lobe. (A) demonstrates the fluid level prior to referral; (B) taken in 1962

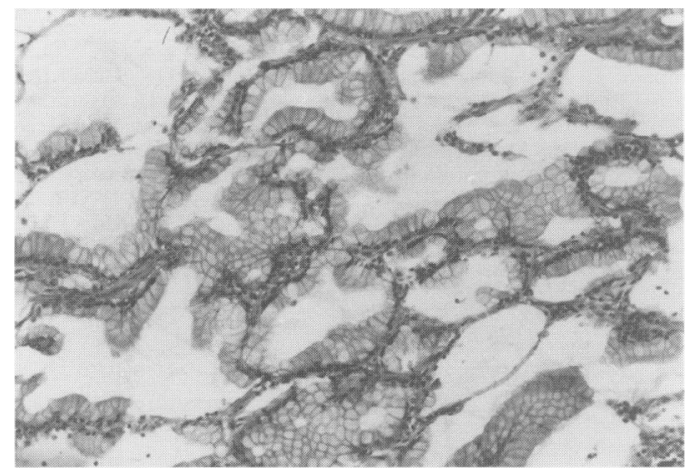

Questions

1 What was the diagnosis?

2 What other diagnoses should be considered?

Figure 2 Histology - see text for details 


\section{Answers}

QUESTION 1

The histology is consistent with a bronchioloalveolar cell carcinoma (BAC). Microscopy shows a tumour composed of well-differentiated mucin-containing columnar cells that line the respiratory spaces without invading the stroma. Mucin stains (not shown) confirmed the presence of mucin within the alveoli and apices of columnar cells.

\section{QUESTION 2}

The presence of a peripheral well-differentiated adenocarcinoma infiltrating along alveolar septa with an intact interstitial framework of the lung differentiates between BAC and nonBAC adenocarcinoma. It is necessary to exclude metastasis from a mucus-secreting adenocarcinoma at an extrapulmonary site (eg, pancreas) as a cause of the tumour, because this will give the same histological pattern which cannot be differentiated from that of a primary lung tumour.

\section{Discussion}

Long-standing lung cysts are often presumed to be congenital in origin. Complications of these cysts include infection (which can distort histological features making the distinction between congenital and acquired aetiology impossible), haemorrhage, enlargement and consequent compression of lung tissue, rupture with pneumothorax, and squamous or adenocarcinoma. Only rarely, as in the case presented, has a BAC been described in association with a long-standing lung-cyst. ${ }^{1,2}$

\begin{tabular}{|l|}
\hline Summary/learning points \\
\hline - malignant change can occur in long-standing \\
lung cysts \\
- clinical radiological changes should prompt \\
early investigation to exclude an occult \\
carcinoma \\
- non invasive procedures for diagnosis can be \\
unreliable \\
- there should be a low threshold for early \\
surgical resection of long-standing solitary lung \\
cysts \\
\hline
\end{tabular}

1 Pritchard MG, Brown PJE, Sterrett GF. Bronchioloalveolar carcinoma arising in longstanding lung cysts. Thorax 1984; 39: 545-9.

2 Hurley P, Corbishley C, Pepper J. Bronchiolo-alveolar carcinoma arising in longstanding lung cysts. Thorax 1985; 40: 960 .

3 Barsky SH, Cameron R, Osann KE, Tomita D, Holmes EC. Rising incidence of bronchiolo-alveolar lung carcinoma and its unique clinicopathologic features. Cancer 1994; 73: its unique

4 Hsu C-P, Chen C-Y, Hsu N-Y. Bronchiolo-alveolar 4 Hsu C-P, Chen C-Y, Hsu N-Y. Bronchiolo-alveolar
carcinoma. f Thorac Cardiovasc Surg 1995; 110: 374-81.
BAC is usually classified as a form of adenocarcinoma, although some consider it a distinct clinicopathological entity due to its different distribution and behaviour. ${ }^{3}$ It represents less than $5 \%$ of lung cancers although some authors have reported a rising incidence. ${ }^{3}$ It occurs more frequently in young patients and in areas of local pulmonary scaring, while there are no sex differences and there is no definite link with smoking. Noninvasive procedures for diagnosis are unreliable ${ }^{4}$ (in this review $80 \%$ of diagnoses were made following operative resection/biopsy and $<10 \%$ by cytological examination). It can present as a solitary, multicentric, or diffuse lesion and has mucinous (associated with a poorer prognosis) and non-mucinous forms. Metastasis occurs via lymphatic and haematogenous routes and rapid endobronchial spread ${ }^{5}$ has been reported in a few patients who presented with localised lesions.

It has been suggested that an unstable epithelial component within the wall of a congenital cyst may be responsible for the later development of a carcinoma. ${ }^{6}$ Womack $^{7}$ in 1941 described metaplasia occurring in the wall of congenital cysts and suggested it may be a precursor of malignancy. More recently Sheffield ${ }^{8}$ noted mucinous epithelial hyperplasia in two young infants and found a mucinous-type BAC in an 18-year-old, all of whom had undergone surgical removal of a cystic congenital adenomatoid malformation (a type of congenital cyst where there has been proliferation of terminal bronchioles at the expense of alveoli). He hypothesised that a premalignant change might occur years prior to the development of a carcinoma in these patients. The cases of BAC occurring in association with longstanding cysts have also been of the mucinous type.

Symptoms from a previously untroublesome longstanding lung cyst may thus indicate malignant change, which may not be detected by noninvasive investigations. Early surgical excision of such cysts should be considered.

\section{Final diagnosis}

Bronchiolo-alveolar carcinoma.

Keywords: bronchiolo-alveolar carcinoma, lung cyst

5 Ferraro L, Solis R, Khan MA. Endobronchial spread of bronchiolo-alveolar cell carcinoma. Chest 1994; 105: 1627-

6 Benjamin DR, Cahill JL. Bronchiolo-alveolar carcinoma of the lung and congenital cystic adenomatoid malformation. Am 7 Clin Pathol 1991; 95: 889-92.

7 Womack NA, Graham EA. Epithelial metaplasia in congenital cystic disease of the lung. Am $\mathcal{f}$ Pathol 1941; 17: genital cysti

8 Sheffield EA, Addis BJ, Corrin B, McCabe MM. Epithelial hyperplasia and malignant change in congenital lung cysts. $\mathcal{f}$ Clin Pathol 1987; 40: 612-4. 